\title{
Integration of oral health-related quality of life instruments
}

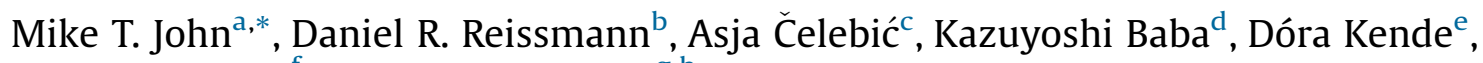 \\ Pernilla Larsson ${ }^{\mathrm{f}}$, Ksenija Rener-Sitar ${ }^{\mathrm{g}, \mathrm{h}}$ \\ ${ }^{a}$ Department of Diagnostic and Biological Sciences, University of Minnesota, 7-536 Moos Tower 515 Delaware Street SE, Minneapolis, MN 55455, USA \\ ${ }^{\mathrm{b}}$ Department of Prosthetic Dentistry, Center for Dental and Oral Medicine, University Medical Center Hamburg-Eppendorf, Hamburg, Germany \\ ${ }^{\mathrm{c}}$ Department of Prosthodontics, University of Zagreb, and School of Dental Medicine and Clinical Hospital Centre, Zagreb, Croatia \\ d Department of Prosthodontics, Showa University, Tokyo, Japan \\ e Department of Prosthodontics, University of Pécs, Pécs, Hungary \\ ${ }^{\mathrm{f}}$ Centre of Oral Rehabilitation, Prosthetic Dentistry, Norrköping, Sweden \\ ${ }^{\mathrm{g}}$ Department of Prosthodontics, University of Ljubljana, Ljubljana, Slovenia \\ ${ }^{\mathrm{h}}$ Department of Prosthodontics, University Dental Clinics, University Medical Center Ljubljana, Ljubljana, Slovenia
}

\section{A R T I C L E I N F O}

\section{Article history:}

Received 18 April 2016

Received in revised form 23 June 2016

Accepted 24 June 2016

\section{Keywords:}

Oral health-related quality of life

Measurement

Questionnaire

Cross-sectional study

Validity

Dimensionality

\begin{abstract}
A B S T R A C T
Objectives: To integrate items from two widely used oral health-related quality of life (OHRQoL) questionnaires, the General Oral Health Assessment Index (GOHAI) and the Oral Impacts on Daily Performances (OIDP), as well as culturally-specific items of the Oral Health Impact Profile (OHIP) into a four-dimensional OHRQoL model consisting of Oral Function,Orofacial Pain, Orofacial Appearance, and Psychosocial Impact.

Methods: Subjects came from an ancillary study of the Dimensions of Oral Health-Related Quality of Life Project ( $N=267$ patients, mean age \pm SD: $54.0 \pm 17.2$ years, 58\% women.) Patients filled in the original 49 items of OHIP and 22 additional OHRQoL items in a cross-sectional study. These additional items consisted of 7 culturally specific OHIP items and 15 GOHAI or OIDP items with unique content not covered in OHIP-49. Before data collection, three experts hypothesized to which of the four OHRQoL dimensions these items belong. Hypotheses were tested in correlation analyses between the 22 items and the four dimension scores that were derived from OHIP-49.

Results: Five of the 22 items did not provide sufficient information to which dimension they belong. In 16 of the remaining 17 items, the pattern of correlation coefficients fitted experts' a priori hypotheses. Acceptance of 16 of the 17 hypotheses was interpreted as evidence that additional (not in OHIP-49 contained) OHRQoL items can be assigned to Oral Function, Orofacial Pain, Orofacial Appearance, and Psychosocial Impact.

Conclusion: Items of three OHRQoL instruments can be integrated into a dimensional OHRQoL model consisting of Oral Function, Orofacial Pain, Orofacial Appearance, and Psychosocial Impact.

Clinical significance: Oral Function, Orofacial Pain, Orofacial Appearance, and Psychosocial Impact can serve as a simple and clinically appealing set of oral health-related quality of life (OHRQoL) dimensions and therefore provide an opportunity for simpler, but psychometrically improved OHRQoL measurement in the future.
\end{abstract}

(c) 2016 Elsevier Ltd. All rights reserved.

\section{Introduction}

Oral health-related quality of life (OHRQoL) is a popular concept to assess the impact of oral conditions and the effects of dental interventions. Many instruments exist to measure OHRQoL. This situation attests to the dental community's interest in OHRQoL, but it also challenges comparability and communication of results.

\footnotetext{
* Corresponding author.

E-mail address: mtjohn@umn.edu (M.T. John).
}

Therefore, from a conceptual point of view, reduction of the number of instruments seems necessary to improve standardization for using OHRQoL assessment globally. In addition, to be confident in the instrument scores, psychometric properties such as reliability, validity, responsiveness, and interpretability need to be known. From a practical point of view, this can only be achieved for a limited number of questionnaires.

However, instead of abandoning some OHRQoL instruments, combining them with other instruments - a synthesis of questionnaires - seems a promising strategy that can achieve better quality instruments while reducing their quantity. Some 
studies have already utilized several OHRQoL instruments together [1-4] and other studies have even submitted them as a combined measure of OHRQoL in analyses [5,6]. However, a more formal assessment whether instruments can be combined has not been performed yet. The prerequisite for a synthesis would be that instruments measure the same underlying latent attribute. Substantial correlations among OHRQoL questionnaire scores attest to this proposition empirically $[2,4,6,7]$. However, these results apply to the OHRQoL global measure, the instrument summary score, but instrument combination needs to be done on the dimension level because these are the attributes that are actually measured and OHRQoL is just the global umbrella term. Conceptually, the hypothesized constructs that are measured by the General Oral Health Assessment Index (GOHAI) and the Oral Impacts on Daily Performances (OIDP) - two frequently used OHRQoL questionnaires - are similar to the four dimensions of OHRQoL (Oral Function, Orofacial Pain, Orofacial Appearance, and Psychosocial Impact) measured by OHIP [8,9], the most widely used OHRQoL instrument. Therefore, OHIP's dimensions may serve as the framework to integrate the other OHRQoL instruments' items. If this proposition would hold, GOHAI and OIDP items as well as the existing culture-specific OHIP items [10-12], i.e., additional indicators of the OHRQOL construct measured with OHIP, should fall into the four OHIP-based dimensions. A relevant target population where such a new grouping of OHRQoL items could be studied would be prosthodontic patients. They could be considered typical dental patients because their number is large, they usually suffer from tooth loss resulting from the two major oral diseases, caries and periodontitis, and several other dental treatments are often performed in conjunction with prosthodontic therapy [13].

Our study aimed to integrate items from two widely used OHRQoL questionnaires, the General Oral Health Assessment Index (GOHAI) and the Oral Impacts on Daily Performances (OIDP), as well as culturally specific items of the Oral Health Impact Profile (OHIP) into a four-dimensional OHRQoL model consisting of Oral Function, Orofacial Pain, Orofacial Appearance, and Psychosocial Impact.

\section{Methods}

\subsection{Study setting, study design, and subjects}

This is an ancillary study initiated within the Dimensions of Oral Health-Related Quality of Life (DOQ) Project [13]. The DOQ Project aims to identify OHIP's dimensional structure, i.e., how many important components the multidimensional oral health perception has. The project analyzes 49-item OHIP [14] data from general population subjects and prosthodontic patients from six countries (Croatia, Germany, Hungary, Japan, Slovenia, and Sweden) with validated OHIP instruments [11,12,15-18]. The international collaborators of the DOQ Project came from the Department of Prosthodontics, University of Zagreb, Croatia; the Department of Prosthetic Dentistry, University Medical Center Hamburg-Eppendorf, Germany; the Department of Prosthodontics, University of Pécs, Hungary; the Department of Prosthodontics, Showa University, Japan; the Department of Prosthetic Dentistry, University of Ljubljana, Slovenia and the Centre of Oral Rehabilitation, Prosthetic Dentistry, Norrköping, Sweden. In each participating center, we targeted a consecutive sample of prosthodontic patients to study a new, one-week recall period for OHIP in a cross-sectional study [19]. In this study, patients were assessed on two occasions when their OHRQoL was assumed to be stable. Specifically, they were assessed either twice before the start of prosthodontic treatment or twice after the end of treatment. On average, two weeks elapsed between assessments. The order in which patients completed the two forms was determined by random assignment. The study design is similar to test-retest studies that were performed for testing the psychometric properties for language-specific OHIP versions in these six countries. However, instead of receiving two OHIPs with the same recall period, one OHIP form had a new, 7-day recall period and the other OHIP form had the commonly used onemonth recall period. For the present study, only the OHIP data with the one-month recall period were used. For details see our previous publication [19]. Institutional Review Boards reviewed and approved the studies in Croatia (Ethical Board of the School of Dental Medicine, University of Zagreb, 05-PA-26-55/06, PDS-103/ 09-10), Hungary (Ethics Committee of the University of Pecs Faculty of Medicine, 2009-3562), Germany (Institutional Review Board of the Medical Association in Hamburg, Germany, PV3530), Japan (Ethics Committees of Showa University, \#2007-29), Slovenia (National Medical Ethic Committee of the Republic of Slovenia, 124/02/13), and Sweden (Regional Ethics Review Board at Linköping University Hospital, 72/04, M208-07).

\subsection{Data analysis}

We studied the most popular OHRQoL instruments, i.e., OHIP, GOHAI, and OIDP. The OHIP-49 served to identify OHRQoL dimensions. Seven additional, culture-specific OHIP items, 15 GOHAI and 12 OIDP items were investigated whether they fit into the OHRQoL dimensions. Before the study, three experts (KRS, DRR, MTJ) independently assessed the GOHAI and the OIDP item content's overlap with OHIP to minimize patients' burden and to improve compliance by avoiding the use of very similar items. Then, in a discussion the experts reached a consensus. The experts also separated themes in GOHAI and OIDP items when they appeared together in a particular question. For example, a GOHAI question asks 'How often did you have trouble biting or chewing any kinds of food, such as firm meat or apples?' Because OHIP asks 'Have you had difficulty chewing any foods because of problems with your teeth, mouth, jaw or dentures?', only 'biting' was selected as a separate theme/problem to be retained in a new item with unique content. Taking culture-specific and original OHIP items' content into account, 12 GOHAI-related items, and then three OIDP-related items had unique content. Together with the seven culture-specific OHIP items, they constituted a set of 22 OHRQoL items with additional, not in OHIP-49 captured OHRQoL content.

To avoid the influence of methods effects, we adapted GOHAI and OIDP items to the OHIP framework and its recall period. For example, the previous GOHAI item was adapted to 'Have you had difficulty biting any foods because of problems with your teeth, mouth, jaw or dentures in the last month?' Available language versions of the GOHAI $[1,4,20,21]$ and the OIDP [22-24] were used to guide the adaptation process. Then, the three experts assigned the 22 items to the four OHIP-based dimensions of Oral Function, Orofacial Pain, Orofacial Appearance, and Psychosocial Impact. Reproducibility of this method was investigated before and found to be adequate [25]. First, the experts did this individually and then synthesized findings by consensus. For most items, assignment as a functional, painful, aesthetical or psychosocial problem was not difficult. Items the experts could not clearly classify into one of the four dimensions were assigned to Psychosocial Impact, because, in their opinion, this dimension represented the broader, more general impact from oral conditions. An example would be the culture-specific OHIP item 'biting cheeks' where the experts could not decide whether it was a functional or a painful problem. This procedure generated 22 hypotheses about OHRQoL items belonging to one of the four OHRQoL dimensions.

Based on the results from the DOQ Project [8], as a marker for these dimensions, we calculated a dimension score 
averaging the items per dimension so that the dimension score would be on the same $0-4$ scale as the 22 item responses. Then, we correlated dimension scores and item responses using a Spearman rank correlation coefficient, resulting in four coefficients per item.

We performed the item-to-dimension classification similarly as in exploratory factor analysis (EFA), except for the difference that in EFA the latent dimensions are not known; in our study, we had four already existing dimensions. In EFA, after extraction of a certain number of unknown factors, the items' correlations (loadings) with these factors are inspected to inform about the dimensions. Only salient items, i.e., items exceeding a certain threshold, are further considered because only they are considered informative. We used in a previous EFA a criterion of 0.45 as a threshold for saliency [9]. Therefore, in the present study only items with a correlation of $\geq 0.45$ were considered informative about a certain dimension and included in hypothesis testing. The item's highest value among the correlations with each of the four dimensions was considered an indication to which dimension this item belonged. When databased and hypothesis-based dimensional assignment agreed, our hypothesis was proved and accepted. Overall, to accept or reject our overarching goal that the set of items from the two OHRQoL instruments and the additional OHIP items can be integrated into the four dimensions, we expected that $75 \%$ of our individual hypotheses would be accepted.

\section{Results}

\subsection{Characterization of prosthodontic patients in six countries}

Sociodemographic and clinical characteristics of the 267 adult patients from six international prosthodontics treatment centers varied substantially (Table 1 ). Overall, patients' mean age was 54.0 years. Fifty-eight percent of the patients were women. The majority of patients had a combination of fixed prosthodontics and their own natural teeth (57\%), but numbers varied from $18 \%$ in Japan, up to $96 \%$ in Slovenia. About half of the study's total patients were assessed after treatment, with Slovenia having the lowest number (32\%) and Japan having the highest (68\%). OHRQoL impairment differed across countries, from a minimum of 18.2 OHIP points in Sweden up to 49.8 OHIP points in Germany (average of 37.2 OHIP points). These differences in OHRQoL impairment across centers were clinically relevant as compared to OHIP-49's Minimal Important Difference of six points [26]. When translated into an absolute problem frequency according to Reissmann et al. [27], between nine (Sweden) and 25 (Germany) impacts per day over the last month occurred in the six treatment centers.

\subsection{Hypotheses testing}

Five items did not reach the threshold for salient correlations (Table 2). 'Swallow comfortably' had very low correlations across dimensions. 'Avoid laughing' had low correlations of similar magnitude to Oral Function, Orofacial Appearance, and Psychosocial Impact and, as expected, a very low correlation with Orofacial Pain. 'Joint noises', 'dry mouth', and 'biting cheeks' correlated rather equally, but low with all four dimensions.

For the 17 remaining items, one hypothesis was rejected. The item 'eating in front of people' was hypothesized to belong to Psychosocial Impact. It correlated substantially with this dimension $(r=0.60)$ but even slightly higher with Oral Function $(r=0.62)$. For 16 items, the highest correlation was in the predicted dimension and consequently, 16 hypotheses were accepted. For two of these 16 correlations, equally high correlation values occurred in another dimension. 'Eating with others' correlated with Psychosocial Impact (predicted) but also with Oral Function. 'Medication for pain' correlated with Psychosocial Impact (predicted) but equally with Orofacial Pain.

Overall, because 16 out of 17 individual hypotheses were accepted, we also accepted our overarching hypothesis that the set of OHRQoL items, having a unique content when compared to OHIP-49, can be assigned to four OHRQoL dimensions.

Ten items belonging to Oral Function, Orofacial Pain, and Orofacial Appearance had their second highest correlation always with Psychosocial Impact. In contrast, when the highest correlation for six items was observed to be Psychosocial Impact, the second highest correlation was evenly distributed among the other three dimensions.

\section{Discussion}

Items from two widely used OHRQoL questionnaires, the GOHAI and the OIDP, as well as culture-specific OHIP items could be integrated into a 4-dimensional OHRQoL model that is based on the most widely used OHRQoL questionnaire, the OHIP. Findings indicate the four OHIP dimensions of Oral Function, Orofacial Pain, Orofacial Appearance, and Psychosocial Impact seem to be generalizable to other OHRQoL questionnaires.

\subsection{Indirect effects of oral conditions}

Furthermore, a pattern emerged, that for indicators of Oral Function, Orofacial Pain, and Orofacial Appearance, the second highest correlation observed was always for Psychosocial Impact. This finding might be interpreted as evidence for indirect effects from oral conditions that we previously hypothesized [28]. Whereas most oral conditions have a primary effect on one of

Table 1

Sociodemographic characteristics, denture status, proportion of follow-up assessments, and OHRQoL impairment in prosthodontics patients of six countries.

\begin{tabular}{|c|c|c|c|c|c|c|c|c|}
\hline & & $\begin{array}{l}\text { All } \\
N=267\end{array}$ & $\begin{array}{l}\text { Croatia } \\
\mathrm{N}=59\end{array}$ & $\begin{array}{l}\text { Germany } \\
\mathrm{N}=37\end{array}$ & $\begin{array}{l}\text { Hungary } \\
\mathrm{N}=49\end{array}$ & $\begin{array}{l}\text { Japan } \\
\mathrm{N}=50\end{array}$ & $\begin{array}{l}\text { Slovenia } \\
\mathrm{N}=50\end{array}$ & $\begin{array}{l}\text { Sweden } \\
\mathrm{N}=22\end{array}$ \\
\hline Females [\%] & & 58.4 & 59.3 & 54.1 & 63.3 & 56.0 & 58.0 & 59.1 \\
\hline Age (SD)[years] & & $54.0(17.2)$ & $62.3(13.2)$ & $54.5(13.8)$ & $46.8(15.4)$ & $68.6(8.7)$ & $40.0(16.1)$ & $46.0(15.4)$ \\
\hline \multirow[t]{4}{*}{ Removable denture status [\% (N)]: } & No removable & $56.6(151)$ & $47.5(28)$ & $48.6(18)$ & $67.3(33)$ & $18.0(9)$ & $96.0(48)$ & $68.2(15)$ \\
\hline & One removable & $35.2(94)$ & $37.3(22)$ & $40.5(15)$ & $26.5(13)$ & $80.0(40)$ & $4.0(2)$ & $9.1(2)$ \\
\hline & Two complete & $6.0(16)$ & $15.3(9)$ & $10.8(4)$ & $4.1(2)$ & $2.0(1)$ & $0.0(0)$ & $0.0(0)$ \\
\hline & Unknown & $2.2(6)$ & $0.0(0)$ & $0.0(0)$ & $2.0(1)$ & $0.0(0)$ & $0.0(0)$ & $22.7(5)$ \\
\hline \multicolumn{2}{|l|}{ Post-treatment assessments [\%] } & 51.3 & 47.5 & 64.9 & 51.0 & 68.0 & 32.0 & 45.4 \\
\hline \multicolumn{2}{|c|}{ OHIP summary score with a one-month recall period [mean (SD)] } & $34.9(29.2)$ & $40.3(27.8)$ & $46.3(42.1)$ & $32.1(30.6)$ & $36.6(23.3)$ & $28.8(20.9)$ & $16.9(21.6)$ \\
\hline
\end{tabular}


Table 2

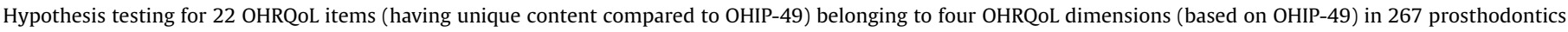
patients from six countries.

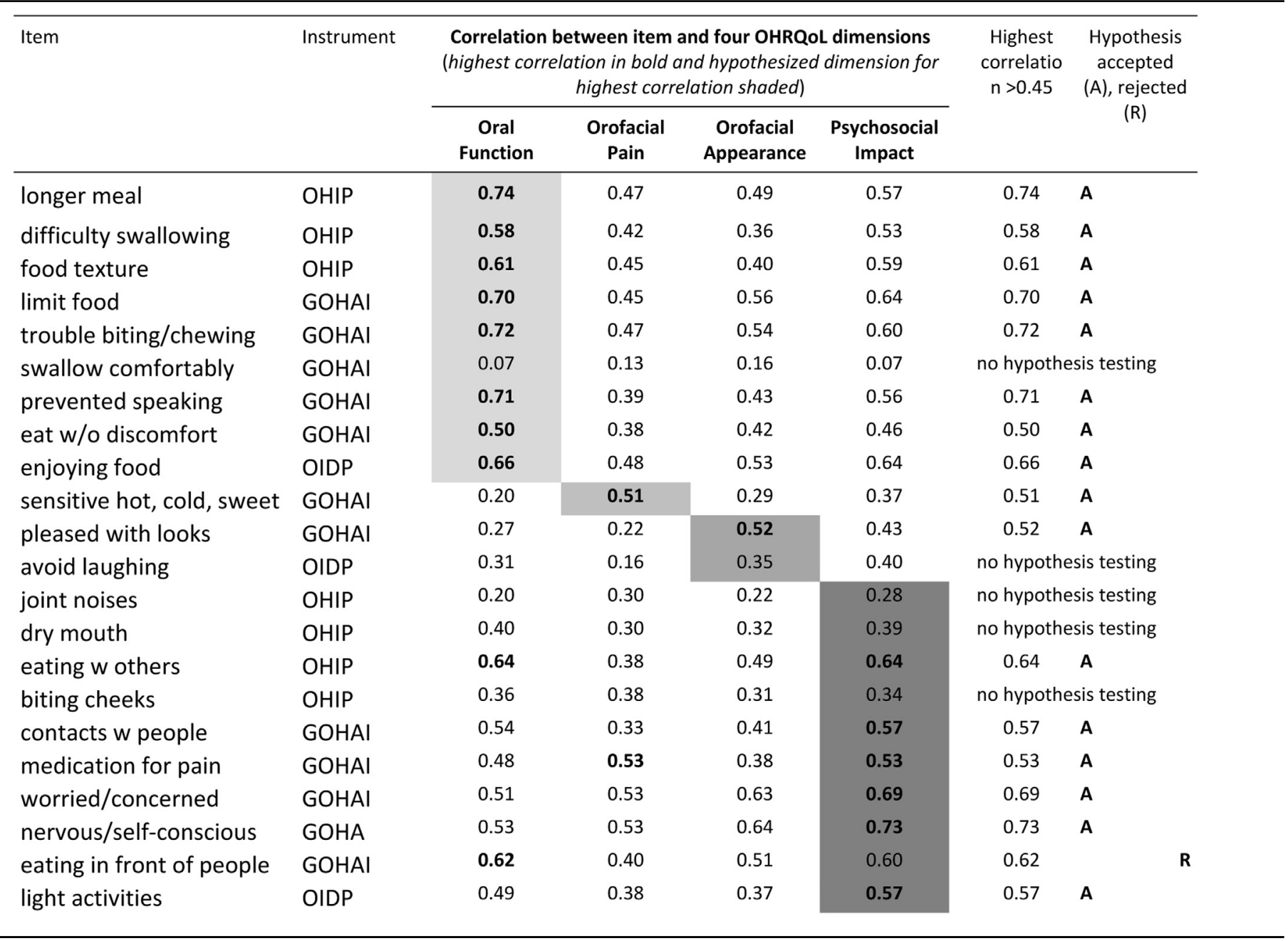

those three dimensions Oral Function, Orofacial Pain, and Orofacial Appearance (e.g., TMD on Orofacial Pain, tooth loss on Oral Function and/or Orofacial Appearance), conditions are often associated with a substantial psychosocial influence, i.e., they have simultaneous or, more likely, subsequent Psychosocial Impact. Several authors have already proposed a stepwise (dimension to dimension) hierarchical framework for oral health impacts. Locker proposed that oral diseases can lead to impairment (a structural condition), further leading to Functional Limitations, Pain/Discomfort and Handicap. In his model, Disability is an intermediate stage in the transition from less to more impaired perceived oral health, with Handicap being the final stage [29]. Cross-sectional data from the general population were later interpreted as support for this model with Functional Limitation, as well as Pain and Discomfort as a first, Disability as a second, and Handicap as a third level of oral health impact [30]. While our findings share similarities with the previous study, they are more in line with a framework presented for the OIDP [31]. These authors examined hypothesized Pain, Discomfort, Functional Limitation, and Dissatisfaction with Appearance as "Level 2 Intermediate Impacts" (Level 1 is Impairment) and Physical, Psychological and Social Performances as "Level 3 Intermediate Impacts". We believe that this sequence - that primary functional, painful, and aesthetical problems have subsequent psychosocial impacts - represents a typical situation when the primary influence is strong. While this is a frequent scenario, an isolated primary influence on Psychosocial Impact can also occur, as demonstrated before [28].

\subsection{OHRQoL dimensions}

That OHIP-based dimensions are applicable to other instruments is not surprising. All OHRQoL instruments measure the same construct. Consequently, instrument scores should correlate highly. In fact, pairwise correlations between OHIP, GOHAI, and OIDP's total scores provided empirical evidence for an overlap in content. For example, GOHAI and OHIP-14 correlated highly, with a value of -0.83 in Swedish dental patients [4], -0.76 in elderly Germans [1], and 0.73 in elderly Japanese [2] (direction of the correlation differed depending on coding of scores). OIDP and OHIP-14 also correlated highly, with a value of 0.78 in British dental hospital patients [7] and with 0.76 in Brazilian postpartum women [3]. When the three OHRQoL instruments and a fourth questionnaire (Oral Health Quality of Life-UK, OHQoL-UK@ [32]) were studied to determine the association between tooth loss - oral health's major physical health indicator - and OHRQoL, an association was present and seemed to be independent from the specific OHRQoL instrument used [33]. However, evidence that the measures correlated well with their dimensions is not available. Methodological reasons may be responsible for this situation. While factor analyses have been performed for OHIP-14, GOHAI, 
and OIDP, they have consistently missed GOHAI's hypothesized dimensions of Physical Function, Psychosocial Function, and Pain/ Discomfort [34], as well as OIDP's hypothesized dimensions Pain, Discomfort, Functional Limitation, Dissatisfaction with Appearance, and Physical, Psychological and Social Performances [31]. However, authors performed factor analyses using OHIP in conjunction with GOHAI or with OIDP $[5,6]$. This indicated that authors thought that OHRQoL items/instruments are compatible enough to be combined. Resulting findings for OHIP-OIDP identified Functional Limitations, Pain-Discomfort, and Psychosocial Impacts as dimensions - identical to the four-dimensional OHRQOL (based on OHIP), except for Orofacial Appearance. We believe that Orofacial Appearance is so important for individuals - on par with Orofacial Pain and Oral Function - that this component of perceived oral health deserves a place in the theoretical structure of OHRQoL. For the GOHAI-OHIP analysis, a comparison is not so straightforward because six factors were found but not named in the study [6].

Our findings corroborate previous results well. As previously mentioned, items did not only correlate highly with the predicted dimension but also notably with other dimensions. This was observed before [8] and is an indication that a strong general factor underlies OHRQoL, and that this construct can probably best be described by a hierarchical model with four first-order factors (dimensions) and a higher-order, general factor model [8]. Findings are also in line with previous findings [9] that not all existing OHRQoL indicators (items) are well suited to be incorporated into OHRQoL dimensions. Methodological reasons are partly responsible. For example, the OHIP item 'Have dental problems made you miserable' missed previously with 0.42 our threshold for a salient correlation (loading) and was not included in the Psychosocial Impact dimension even if this item seems to be a very plausible indicator. The same situation may apply to the item 'avoid laughing' in the current study. Based on the OHIP item 'avoid smiling' being a strong indicator for Orofacial Appearance, 'avoid laughing' had a substantial correlation with this dimension but also with Psychosocial Impact. More important than methodological influences is probably the fact that items such as 'joint noises' and 'dry mouth' are symptoms of oral conditions. The presence of symptoms in OHIP was previously observed for the OHIP item 'stale breath,' which could not be assigned to one of the four dimensions [9]. Conceptually, symptoms of oral diseases should not be incorporated in a dimensional OHRQoL model. Finally, we also encountered problems with some items. The item 'swallow comfortably' did not show an interpretable pattern of correlations, whereas an item with similar content ('difficulty swallowing') did. This indicates that OHRQoL items will still need more methodological work regarding how they perform across cultures.

Overall, while the findings of a strong general OHRQoL factor and indirect effects from one dimension to another are relevant from a conceptual point of view to elucidate mechanisms of oral conditions' influences on individuals, they also point to technical challenges. Items having a considerable correlation with more than one dimension will make dimensions more similar. This situation challenges the usefulness of dimension scores. Further item analyses are necessary, leading to the deletion of certain items and the addition of more specific items, to refine OHRQoL dimensions. Alignment of oral health with health-related quality of life measurement is also recommended [35].

\subsection{Strengths and limitations}

For studies using questionnaires, it should be verified that scores are valid and reliable in the target population. While the OHIP-49 has documented sufficient psychometric properties in all six study countries [11,12,15-18], specific GOHAI $[1,4,20,21]$ and OIDP [22-24] language versions and the extra OHIP questions were only available for some settings. We did not perform a rigorous cross-cultural adaptation, which is a limitation. However, we believe that the introduced measurement error would probably have biased findings against the observed pattern. It seems unlikely that translation error and other influences would have moved results closer to a plausible, clinically interpretable pattern of findings.

We performed a correlation analysis to study the research question and we combined data without taking into account that they came from different countries, i.e., our data had a hierarchical structure. More sophisticated analyses exist, e.g., factor analysis, but these statistical techniques require more subjects than available. On the other hand, researchers have used other techniques besides factor analysis before $[25,30]$ to investigate dimensionality. Our robust correlation analysis, not relying on many statistical assumptions, provided a sound and interpretable pattern of findings. Finally, we studied GOHAI and OIDP. While these two instruments are the two most widely used OHRQoL instruments besides the OHIP, many other instruments exist [36]. All the instruments share many similarities and the items of the two we studied should be seen as a sample of OHRQoL indicators in general.

\section{Conclusion}

The study advances our theory of oral health. While OHIP-based dimensions could be generalized to OHRQoL dimensions based on conceptual reasons before, we now have more evidence that Oral Function, Orofacial Pain, Orofacial Appearance, and Psychosocial Impact can serve as a simple and clinically meaningful set of OHRQoL dimensions because several instruments' items fit into these dimensions.

The study also points to future practical improvement of OHRQoL measurement. While short instruments that are based on the four-dimensional OHRQoL model such as the 5-item OHIP [37] already exist and were validated [38], OHRQoL measurement can be advanced. The many existing OHRQoL instruments can integrate their items into the dimensions. Only four instruments - one for each OHRQoL dimension - are necessary to comprehensively measure the major patient-perceived influences from oral disorders and dental interventions.

\section{Conflicts of interest}

None.

\section{Acknowledgements}

Research reported in this publication was supported by the National Institute of Dental and Craniofacial Research of the National Institutes of Health under Award Number R01DE022331.

\section{Appendix A. Supplementary data}

Supplementary data associated with this article can be found, in the online version, at http://dx.doi.org/10.1016/j.jdent.2016. 06.006.

\section{References}

[1] A.J. Hassel, C. Rolko, U. Koke, J. Leisen, P. Rammelsberg, A German version of the GOHAI, Community Dent. Oral Epidemiol. 36 (2008) 34-42.

[2] K. Ikebe, T. Hazeyama, K. Enoki, S. Murai, T. Okada, R. Kagawa, et al. Comparison of GOHAI and OHIP-14 measures in relation to objective values of oral function in elderly Japanese, Community Dent. Oral Epidemiol. 40 (2012) 406-414. 
[3] B.H. Oliveira, P. Nadanovsky, Psychometric properties of the Brazilian version of the oral health impact profile-short form, Community Dent. Oral Epidemiol. 33 (2005) 307-314.

[4] C. Hagglin, U. Berggren, J. Lundgren, A Swedish version of the GOHAI index: psychometric properties and validation, Swedish Dental Journal 29 (2005) 113-124.

[5] J. Montero, M. Bravo, M.P. Vicente, M.P. Galindo, J.F. Lopez, A. Albaladejo, Dimensional structure of the oral health-related quality of life in healthy Spanish workers, Health Qual. Life Outcomes 8 (2010) 24.

[6] A.J. Hassel, B. Steuker, C. Rolko, L. Keller, P. Rammelsberg, I. Nitschke, Ora health-related quality of life of elderly Germans-comparison of GOHAI and OHIP-14, Community Dent. Health 27 (2010) 242-247.

[7] P.G. Robinson, B. Gibson, F.A. Khan, W. Birnbaum, Validity of two oral healthrelated quality of life measures, Community Dent. Oral Epidemiol. 31 (2003) 90-99.

[8] M.T. John, L. Feuerstahler, N. Waller, K. Baba, P. Larsson, A. Celebic, et al., Confirmatory factor analysis of the oral health impact profile, J. Oral Rehabil. 41 (2014) 644-652.

[9] M.T. John, D.R. Reissmann, L. Feuerstahler, N. Waller, K. Baba, P. Larsson, et al. Exploratory factor analysis of the oral health impact profile, J. Oral Rehabil. 41 (2014) 635-643.

[10] H. Murray, D. Locker, D. Mock, H.C. Tenenbaum, Pain and the quality of life in patients referred to a craniofacial pain unit, J. Orofac. Pain 10 (1996) 316-323.

[11] M.T. John, D.L. Patrick, G.D. Slade, The german version of the oral health impact profile-translation and psychometric properties, Eur. J. Oral Sci. 110 (2002) 425-433.

[12] M. Yamazaki, M. Inukai, K. Baba, M.T. John, Japanese version of the oral health impact profile (OHIP-J), J. Oral Rehabil. 34 (2007) 159-168.

[13] M.T. John, D.R. Reissmann, L. Feuerstahler, N. Waller, K. Baba, P. Larsson, et al, Factor analyses of the oral health impact profile-overview and studied population, J. Prosthodont. Res. 58 (2014) 26-34.

[14] G.D. Slade, A.J. Spencer, Development and evaluation of the oral health impact profile, Community Dent. Health 11 (1994) 3-11.

[15] A. Szentpetery, G. Szabo, G. Marada, I. Szanto, M.T. John, The hungarian version of the oral health impact profile, Eur. J. Oral Sci. 114 (2006) 197-203.

[16] N. Petricevic, A. Celebic, M. Papic, K. Rener-Sitar, The croatian version of the oral health impact profile questionnaire, Coll. Antropol. 33 (2009) 841-847.

[17] K. Rener-Sitar, A. Celebic, N. Petricevic, M. Papic, D. Sapundzhiev, A. Kansky, et al., The slovenian version of the oral health impact profile questionnaire (OHIP-SVN): translation and psychometric properties, Coll. Antropol. 33 (2009) 1177-1183.

[18] P. Larsson, T. List, I. Lundstrom, A. Marcusson, R. Ohrbach, Reliability and validity of a Swedish version of the oral health impact profile (OHIP-S), Acta Odontol. Scand. 62 (2004) 147-152.

[19] N.G. Waller, M.T. John, L. Feuerstahler, K. Baba, P. Larsson, S. Peršić, et al., A 7day recall period for a clinical application of the oral health impact profile questionnaire, Clin. Oral Investig. 20 (2016) 91-99.

[20] M. Naito, Y. Suzukamo, T. Nakayama, N. Hamajima, S. Fukuhara, Linguistic adaptation and validation of the general oral health assessment index (GOHAI) in an elderly Japanese population, J. Public Health Dent. 66 (2006) 273-275.
[21] A. Lomšek, B. Artnik, R. Gašperšič, The influence of socio-demographic factors on self-perception of oral health in periodontal patients, J. Clin. Periodontol. 39 (Suppl. 13) (2012) 157-158.

[22] M. Naito, Y. Suzukamo, H.O. Ito, T. Nakayama, Development of a Japanese version of the oral impacts on daily performance (OIDP) scale: a pilot study, J. Oral Sci. 49 (2007) 259-264.

[23] A.L. Ostberg, P. Andersson, M. Hakeberg, Cross-cultural adaptation and validation of the oral impacts on daily performances (OIDP) in Swedish, Swed. Dent. J. 32 (2008) 187-195.

[24] V. Lajnert, R. Gržić, N. Radica, D. Šnjarić, S. Špalj, Translation and validation of the Croatian version of the oral impacts on daily performances (OIDP) scale, Vojnosanit. Pregl. 73 (2015) 136.

[25] M.T. John, Exploring dimensions of oral health-related quality of life using experts' opinions, Qual. Life Res. 16 (2007) 697-704.

[26] M.T. John, D.R. Reissmann, A. Szentpétery, J.G. Steele, An approach to define clinical significance in prosthodontic patients, J. Prosthodont.18(2009)455-460.

[27] D.R. Reissmann, I. Sierwald, G. Heydecke, M.T. John, Interpreting one oral health impact profile point, Health Qual. Life Outcomes 11 (2013) 12.

[28] M.T. John, K. Rener-Sitar, B. Baba, A. Čelebić, P. Larsson, G. Szabo, et al., Patterns of impaired oral health-related quality of life dimensions, J. Oral Rehabil. 43 (2016) 519-527.

[29] D. Locker, Measuring oral health: a conceptual framework, Community Dent. Health 5 (1988) 3-18.

[30] N. Nuttall, G.D. Slade, A.E. Sanders, J.G. Steele, P. Allen, S. Lahti, An empirically derived population-response model of the short form of the oral health impact profile, Community Dent. Oral Epidemiol. 34 (2006) 18-24.

[31] S. Adulyanon, A. Sheiham, Oral impacts on daily performances, in: G.D. Slade (Ed.), Measuring Oral Health and Quality of Life, University of North Carolina, Department of Dental Ecology, Chapel Hill, 1997, pp. 151-160.

[32] C. McGrath, R. Bedi, An evaluation of a new measure of oral health related quality of life-OHQoL-UK(W), Community Dent. Health 18 (2001) 138-143.

[33] A.E. Gerritsen, P.F. Allen, D.J. Witter, E.M. Bronkhorst, N.H. Creugers, Tooth loss and oral health-related quality of life: a systematic review and meta-analysis, Health Qual. Life Outcomes 8 (2010) 126.

[34] K.A. Atchison, The general oral health assessment index, in: G.D. Slade (Ed.), Measuring Oral Health and Quality of Life, University of North Carolina, Department of Dental Ecology, Chapel Hill, 1997, pp. 71-80.

[35] D.R. Reissmann, Alignment of oral health-related with health-related quality of life assessment, J. Prosthodont. Res, 60 (2016) 69-71.

[36] E. Skaret, A. Nordrehaug Åstrøm, O. Haugejorden, Oral Health-related quality of life (OHRQoL)-review of existing instruments and suggestions for use in oral health outcome research in Europe, in: D.M. Bourggeois, J.C. Llodra (Eds.), Health Surveillance in Europe - European Global Oral Health Indicators Development Project - 2003 Report Proceedings, Quintessence International, Paris, 2004, pp. 99-110.

[37] M.T. John, D.L. Miglioretti, L. LeResche, T.D. Koepsell, P. Hujoel, W. Micheelis, German short forms of the oral health impact profile, Community Dent. Oral Epidemiol. 34 (2006) 277-288.

[38] A. Naik, M.T. John, N. Kohli, K. Self, P. Flynn, Validation of the english-language version of 5-item oral health impact profile, J. Prosthodont. Res. 60 (2016) 85-91. 